03

\title{
Закон сопротивления для турбулентного течения Тейлора-Куэтта при очень больших числах Тейлора
}

\author{
(C) A.M. Балонишников
}

Санкт-Петербургский государственный университет аэрокосмического приборостроения, 190000 Санкт-Петербург, Россия

e-mail: balonishnikov@mail.ru

(Поступило в Редакцию 2 ноября 2015 г.)

Выполнены исходя из полуэмпирической модели переноса удельной скорости диссипации турбулентной энергии законы сопротивления для турбулентного течения Тейлора-Куэтта при вращении как внешнего, так и внутреннего цилиндров при очень больших числах Тейлора.

\section{Введение}

Турбулентное течение жидкости в зазоре между двумя вращающимися коаксиальными цилиндрами является одним из важнейших типов течений для понимания механизмов развитой турбулентности. Основными параметрами такого течения являются: $a-$ радиус внутреннего цилиндра, $b$ - радиус внешнего цилиндра, $\rho-$ плотность жидкости, $v-$ коэффициент молекулярной кинематической вязкости, длина каждого из цилиндров, $\omega_{1}$ - угловая скорость вращения внутреннего цилиндра, $\omega_{2}$ - угловая скорость вращения внешнего цилиндра,

$$
G=T /\left(\rho v^{2} L\right)=2 \pi a^{2} u_{1}^{* 2} / v^{2},
$$

где $G$ - безразмерный и $T-$ размерный моменты сил соответственно, действующих на боковую поверхность внутреннего цилиндра:

$$
T=\rho u_{1}^{* 2} 2 \pi a L a=2 \pi \rho a^{2} L u_{1}^{* 2},
$$

$u_{1}^{*}$-скорость трения на боковой поверхности внутреннего цилиндра.

\section{Законы сопротивления в рамках обобщенной модели локального баланса}

В работе [1] была представлена однопараметрическая модель турбулентности, основанная на приближении локального баланса турбулентной энергии и уравнения переноса удельной скорости диссипации турбулентной энергии. В качестве граничных условий использовались логарифмические профили скорости. Сшивание аналитических решений уравнений модели для турбулентного течения Тейлора-Куэтта с этими профилями определили следующий закон сопротивления типа Прандтля-Кармана:

$$
\begin{array}{r}
4 \pi q u_{1}^{*} \kappa^{-1}\left(q^{4}-1\right) J_{0}+u_{1}^{*} q k^{-1} \ln \left[C u_{1}^{*}(b-a) / v\right] \\
+u_{2}^{*} \kappa^{-1} \ln \left[C u_{2}^{*}(b-a) / \nu\right]=V_{2}+V_{1} q
\end{array}
$$

где $q=b / a \succ 1, a$ и $b-$ радиусы цилиндров, $u_{1}^{*}$ и $u_{2}^{*}-$ скорости трения на поверхности цилиндров, $\kappa=0.4$ и $C=9.5$ - константы Прандтля-Кармана логарифмического профиля средней скорости, $V_{1}$ и $V_{2}-$ линейные скорости вращения точек боковых поверхностей цилиндров, вращающихся в противоположных направлениях (или один из них покоится), предполагается справедливость известного соотношения для турбулентного течения Тейлора-Куэтта: $u_{1}^{*} a=u_{2}^{*} b$, выражающие закон сохранения углового момента:

$$
\begin{aligned}
J_{0}= & \int_{1}^{q} d x\left\{x \csc \left[\pi\left(x^{4}-1\right) /\left(q^{4}-1\right)\right]\right. \\
& -\left(q^{4}-1\right)(4 \pi)^{-1}(x-1)^{-1} \\
& \left.-\left(q^{4}-1\right)(4 \pi)^{-1} q^{-2}(q-x)^{-1}\right\} .
\end{aligned}
$$

В рамках предложенной модели соотношение (2) справедливо для турбулентного течения Тейлора-Куэтта при произвольных угловых скоростях вращения цилиндров $\omega_{1}$ и $\omega_{2}$, а также для произвольных радиусов цилиндров $a$ и $b$, если вблизи поверхностей цилиндров существуют логарифмические профили средней скорости Прандтля-Кармана. Отметим, что в работе [2] получено предельное выражение для закона сопротивления (2) в случае очень больших чисел Рейнольдса для вращения только внутреннего цилиндра $\left(\omega_{2}=0\right.$ и $\left.V_{2}=0\right)$. Однако представляет большой интерес вид законов сопротивления развитого турбулентного течения Тейлора-Куэтта как при вращении только одного из двух цилиндров, так и в случае двух вращающихся соосных цилиндров. Для исследования этого общего случая правую часть уравнения (2) можно заменить выражением $b\left(\omega_{2}+\omega_{1}\right)$, так как

$$
V_{2}+V_{1} q=b \omega_{2}+a \omega_{1} q=b\left(\omega_{2}+\omega_{1}\right)
$$

(при такой записи предполагалось вращение цилиндров в противоположных направлениях, или один из них находился в покое). 
Теперь будем считать, что положительной будет угловая скорость того цилиндра, который вращается с наибольшей по модулю угловой скоростью. Тогда правую часть уравнения (2) следует записать как $b\left|\omega_{2}-\omega_{1}\right|$. Алгебраическое уравнение (1) принимает вид

$$
\begin{gathered}
4 \pi q u_{1}^{*} \kappa^{-1}\left(q^{4}-1\right)^{-1} J_{0}+u_{1}^{*} q \kappa^{-1} \ln \left[C u_{1}^{*}(b-a) / \nu\right] \\
+u_{1}^{*} q^{-1} \kappa^{-1} \ln \left[C u_{1}^{*} q^{-1}(b-a) / \nu\right]=b\left|\omega_{2}-\omega_{1}\right|,
\end{gathered}
$$

где использовано соотношение $u_{2}^{*}=u_{1}^{*} q^{-1}$.

Случай $\omega_{2}=\omega_{1}$ соответствует твердотельному вращению, когда цилиндры вращаются в одну сторону и, конечно, моделью не описываются.

Выведем алгебраическое уравнение, определяющее безразмерный момент силы $G$ от чисел Рейнольдса $\mathrm{Re}_{1}$ и $\operatorname{Re}_{2}$ :

$$
\operatorname{Re}_{1}=\frac{(b-a)\left|\omega_{1}\right| a}{v}, \quad \operatorname{Re}_{2}=\frac{(b-a)\left|\omega_{2}\right| b}{v},
$$

выражая угловые скорости $\omega_{1}, \omega_{2}$ через неотрицательные числа Рейнольдса $\operatorname{Re}_{1}, \operatorname{Re}_{2}$, а скорость трения через величину $G$ :

$$
u_{1}^{*}=\frac{v}{a} \sqrt{\frac{G}{2 \pi}},
$$

опуская очевидные упрощения из соотношения (4) получим искомое алгебраическое уравнение

$$
\begin{aligned}
& 4 \pi q\left(q^{4}-1\right)^{-1} J_{0} \sqrt{\frac{G}{2 \pi}}+q \sqrt{\frac{G}{2 \pi}} \ln \left(C(q-1) \sqrt{\frac{G}{2 \pi}}\right) \\
& +q^{-1} \sqrt{\frac{G}{2 \pi}} \ln \left(C q^{-1}(q-1) \sqrt{\frac{G}{2 \pi}}\right) \\
& =(q-1)^{-1} \kappa\left|q \operatorname{Re}_{1} \operatorname{sign}\left(\omega_{1}\right)-\operatorname{Re}_{2} \operatorname{sign}\left(\omega_{2}\right)\right| .
\end{aligned}
$$

В работе [3] было предложено исследовать этот общий случай, вводя число Тейлора Та следующим выражением:

$$
\mathrm{Ta}=\frac{1}{4} \sigma d^{2}(a+b)^{2}\left(\omega_{1}-\omega_{2}\right)^{2} v^{-2},
$$

где $\sigma=\left(\frac{1+\eta}{2 \sqrt{\eta}}\right)^{4}$, отношение радиусов цилиндров $\eta=\frac{a}{b}$ $=q^{-1}$, ширина канала $d=b-a$.

В качестве зависимой переменной предлагалось использовать безразмерный момент сил трения $G$, действующих со стороны вращающей жидкости на цилиндрическую поверхность внутреннего цилиндра.

В той же работе [3] предложена следующая эмпирическая зависимость:

$$
G \propto \mathrm{Ta}^{0.88}, \quad \text { при } \quad \mathrm{Ta} \rightarrow \infty .
$$

Для сравнения с нашей моделью перепишем модуль разности угловых скоростей вращающихся цилиндров, используя соотношение (6), через число Тейлора Та:

$$
\left|\omega_{1}-\omega_{2}\right|=\sqrt{\frac{4 \mathrm{Ta}}{\sigma}} \frac{v}{d(a+b)} .
$$

Тогда уравнение (4) примет вид

$$
\begin{aligned}
& \frac{4 \pi q}{\kappa\left(q^{4}-1\right)} J_{0} \sqrt{\frac{G}{2 \pi}}+\frac{q}{\kappa} \sqrt{\frac{G}{2 \pi}} \ln \left[C(q-1) \sqrt{\frac{G}{2 \pi}}\right] \\
& +\frac{1}{q \kappa} \sqrt{\frac{G}{2 \pi}} \ln \left[C q^{-1}(q-1) \sqrt{\frac{G}{2 \pi}}\right]=\frac{q}{q^{2}-1} \sqrt{\frac{4 \mathrm{Ta}}{\sigma}},
\end{aligned}
$$

где

$$
\sigma=\left(\frac{1+\eta}{2 \sqrt{\eta}}\right)^{4}=\left(\frac{1+q}{2 \sqrt{q}}\right)^{4} .
$$

Алгебраическое уравнение (9) определяет неявным образом зависимость безразмерного момента сил $G$ от числа Тейлора Та, параметрами которой служат две константы Кармана: $C, \kappa$ и отношение радиусов цилиндров $q$.

Для определения асимптотического поведения момента сил $G$ при стремлении числа Тейлора к бесконечности: Тa $\rightarrow \infty$ поступим так же, как в работе [2] для определения предельного закона сопротивления устремляли число Рейнольдса к бесконечности. Для удобства введем новые переменные:

$$
\mathrm{Ta}_{1}=\sqrt{\frac{4 \mathrm{Ta}}{\sigma}}, \quad G_{1}=\sqrt{\frac{G}{2 \pi}} .
$$

В новых переменных соотношение (9) примет вид

$$
\begin{aligned}
& \frac{4 \pi q}{\kappa\left(q^{4}-1\right)} J_{0} G_{1}+\frac{q}{\kappa} G_{1} \ln \left[G_{1} C(q-1)\right] \\
& \quad+\frac{1}{k q} G_{1} \ln \left[G_{1} C q^{-1}(q-1)\right]=\frac{q}{q^{2}-1} \mathrm{Ta}_{1} .
\end{aligned}
$$

Для дальнейшего анализа перепишем соотношение (10) в следующем виде:

$$
\begin{array}{r}
\frac{4 \pi J_{0}}{\kappa\left(q^{2}+1\right)} G_{1}+\frac{q^{4}-1}{\kappa q^{2}} G_{1} \ln G_{1}+\frac{q^{2}-1}{\kappa} G_{1} \ln [C(q-1)] \\
+\frac{q^{2}-1}{\kappa q^{2}} G_{1} \ln \left[C q^{-1}(q-1)\right]=\mathrm{Ta}_{1} .
\end{array}
$$

Поскольку натуральный логарифм - функция медленно растущая, то в нулевом приближении $G_{1} \propto \mathrm{Ta}_{1}$, при таком поведении второй член в левой части (11) будет ведущим, и мы можем приближенно считать

$$
\frac{q^{4}-1}{\kappa q^{2}} G_{1} \ln G_{1} \approx \mathrm{Ta}_{1} .
$$

Проведем дальнейшее упрощение соотношения (12)

$$
\frac{q^{4}-1}{\kappa q^{2}} G_{1} \ln \mathrm{Ta}_{1} \approx \mathrm{Ta}_{1} .
$$

Откуда:

$$
G_{1}=\frac{\kappa q^{2} \mathrm{Ta}_{1}}{\left(q^{4}-1\right) \ln \mathrm{Ta}_{1}}
$$


Для оправдания сделанных приближений поделим обе части соотношения (11) на величину $\mathrm{Ta}_{1}$ и подставим полученную зависимость (14) в это соотношение. Первый, третий и четвертый члены левой части уравнения устремятся к нулю, как

$$
\frac{1}{\ln \mathrm{Ta}_{1}} \quad \text { при } \quad \mathrm{Ta}_{1} \rightarrow \infty,
$$

второй член устремится к единице, поскольку

$$
\frac{\ln \left(\ln \mathrm{Ta}_{1}\right)}{\ln \mathrm{Ta}_{1}} \rightarrow 0 \quad \text { при } \quad \mathrm{Ta}_{1} \rightarrow \infty,
$$

что и доказывает справедливость асимптотического поведения величины $G_{1}$ в рамках авторской модели турбулентности. Возвращаясь к исходным переменным $G$ и Та, получим

$$
G=\frac{32 \pi \kappa^{2} q^{4} \mathrm{Ta}}{\left(q^{4}-1\right)^{2} \sigma \ln ^{2}(4 \mathrm{Ta} / \sigma)} \quad \text { при } \quad \text { Та } \rightarrow \infty .
$$

Можно также написать асимптотически эквивалентную формулу

$$
G=\frac{32 \pi \kappa^{2} q^{4} \mathrm{Ta}}{\left(q^{4}-1\right)^{2} \sigma \ln ^{2}(\mathrm{Ta})} \quad \text { при } \quad \text { Та } \rightarrow \infty .
$$

Похожие логарифмические поправки с другими степенями логарифмов были предложены в теоретических работах $[4,5]$.

В некоторых экспериментальных работах $[6,7]$ представлена зависимость безразмерного момента сил $G$ от числа Рейнольдса Re. B случае вращения только внутреннего цилиндра используется число $\operatorname{Re}_{1}=\omega_{1} a d / v$. В случае вращения только внешнего цилиндра используется число $\operatorname{Re}_{2}=\omega_{2} b d / \nu$.

Рассмотрим случай вращения только внутреннего цилиндра: $\omega_{2}=0, \omega_{1}=\frac{\mathrm{Re}_{1} v}{a d}$. Тогда из соотношения (4) получаем алгебраическое уравнение, определяющее неявную зависимость безразмерного момента сил $G$ от числа Рейнольдса $\operatorname{Re}_{1}$ :

$$
\begin{gathered}
4 \pi q \kappa^{-1} \sqrt{\frac{G}{2 \pi}}\left(q^{4}-1\right)^{-1} J_{0}+q \sqrt{\frac{G}{2 \pi}} \kappa^{-1} \ln \left[C(q-1) \sqrt{\frac{G}{2 \pi}}\right] \\
+q^{-1} \sqrt{\frac{G}{2 \pi}} \kappa^{-1} \ln \left[C q^{-1}(q-1) \sqrt{\frac{G}{2 \pi}}\right]=\frac{q \operatorname{Re}_{1}}{(q-1)} .
\end{gathered}
$$

Для упрощения этого выражения используем ранее введенную переменную $G_{1}$ вместо переменной $G$ :

$$
\begin{aligned}
\frac{4 \pi q J_{0}}{q^{4}-1} G_{1} & +G_{1} q \ln \left[C(q-1) G_{1}\right] \\
& +G_{1} q^{-1} \ln \left[C G_{1} q^{-1}(q-1)\right]=\frac{\kappa q \operatorname{Re}_{1}}{q-1} .
\end{aligned}
$$

Найдем асимптотику зависимости $G_{1}=G\left(\operatorname{Re}_{1}\right)$ при $\operatorname{Re}_{1} \rightarrow \infty$. Из уравнения (18) видно, что в нулевом приближении $G_{1} \propto \operatorname{Re}_{1}$. В этом случае ведущим членом в левой части выражения будет $G_{1}\left(q+q^{-1}\right) \ln G_{1}$, что позволяет написать приближенное равенство

$$
G_{1}\left(q+q^{-1}\right) \ln G_{1}=\frac{\kappa q \operatorname{Re}_{1}}{q-1} .
$$

Учитывая приближенную прямую пропорциональность $G_{1} \propto \mathrm{Re}_{1}$, заменим в соотношении (19) $\ln G_{1}=\ln \mathrm{Re}_{1}$, что даст искомую зависимость

$$
G_{1}=\frac{\kappa q^{2} \operatorname{Re}_{1}}{(q-1)\left(q^{2}+1\right) \ln \mathrm{Re}_{1}} .
$$

Так же как это было проделано при выводе соотношения (15), можно убедиться, что выражение (20) есть асимптотика зависимости $G_{1}=G_{1}\left(\operatorname{Re}_{1}\right)$, определяемой алгебраическим выражением (18). Возвращаясь к исходной переменной $G$, получим окончательное выражение

$$
G=\frac{2 \pi \kappa^{2} q^{4} \operatorname{Re}_{1}^{2}}{(q-1)^{2}\left(q^{2}+1\right)^{2} \ln ^{2} \operatorname{Re}_{1}} \quad \text { при } \quad \operatorname{Re}_{1} \rightarrow \infty,
$$

где $G$ - безразмерный момент сил, действующий на вращающийся внутренний цилиндр (внешний цилиндр покоится), $\operatorname{Re}_{1}$ - число Рейнольдса, $q$ - отношение радиуса внешнего цилиндра к радиусу внутреннего цилиндра, $\kappa=0.4$ - константа Кармана.

Рассмотрим случай вращения только внешнего цилиндра (внутренний цилиндр покоится $\left.\omega_{1}=0\right), \omega_{2}=\frac{\mathrm{Re}_{2} v}{b d}$. Тогда из уравнения (4) получим алгебраическое уравнение, определяющее неявным образом зависимость безразмерного момента сил $G$ от числа Рейнольдса $\mathrm{Re}_{2}$ :

$$
\begin{aligned}
& \frac{4 \pi q J_{0}}{q^{4}-1} \sqrt{\frac{G}{2 \pi}}+q \sqrt{\frac{G}{2 \pi}} \ln \left[C(q-1) \sqrt{\frac{G}{2 \pi}}\right] \\
& +q^{-1} \sqrt{\frac{G}{2 \pi}} \ln \left[C q^{-1}(q-1) \sqrt{\frac{G}{2 \pi}}\right]=\frac{\kappa \mathrm{Re}_{2}}{(q-1)} .
\end{aligned}
$$

Рассуждая, как и в случае вращения только внутреннего цилиндра, опустив промежуточные выкладки, получим окончательный предельный закон сопротивления для безразмерного момента сил $G$, действующего, как и в выражении (21), на внутренний цилиндр

$$
G=\frac{2 \pi \kappa^{2} q^{2} \operatorname{Re}_{2}^{2}}{(q-1)^{2}\left(q^{2}+1\right)^{2} \ln ^{2} \operatorname{Re}_{2}} \quad \text { при } \quad \operatorname{Re}_{2} \rightarrow \infty .
$$

Отметим, что в промежуточном диапазоне чисел Рейнольдса и Тейлора для расчета законов сопротивления следует использовать исходные законы сопротивления (9), (17), (22).

Иногда в качестве зависимой переменной в законах сопротивления используют квазичисло Нуссельта $\mathrm{Nu}_{\omega}$,

$$
\mathrm{Nu}_{\omega}=G / G_{l a m}
$$


где $G_{\text {lam }}-$ безразмерный момент силы, действующий на внутренний цилиндр со стороны жидкости при ламинарном движении, согласно [6],

$$
G_{\text {lam }}=2 v^{-1} a^{2} b^{2}\left|\omega_{1}-\omega_{2}\right|\left(b^{2}-a^{2}\right)^{-1} .
$$

Учитывая соотношения (5) и (25), мы можем связать $G_{\text {lam }}$ с числом Тейлора Та:

$$
G_{\text {lam }}=\frac{4 q^{2} \sqrt{\mathrm{Ta}}}{\sqrt{\sigma}\left(q^{2}-1\right)^{2}} .
$$

Комбинируя соотношения (24) и (26), получим

$$
\mathrm{Nu}_{\omega}=\frac{G \sqrt{\sigma}\left(q^{2}-1\right)^{2}}{4 q^{2} \sqrt{\mathrm{Ta}}} .
$$

Используя асимптотическую формулу (16), получим окончательную формулу для квазичисла Нуссельта

$$
\mathrm{Nu}_{\omega}=\frac{8 \pi \kappa^{2} q^{2} \sqrt{\mathrm{Ta}}}{\left(q^{2}+1\right)^{2} \sqrt{\sigma} \ln ^{2} \mathrm{Ta}} \quad \text { при } \quad \mathrm{Ta} \rightarrow \infty .
$$

Отметим, что иногда вместо числа Тейлора Та, используют число Рейнольдса Re:

$$
\operatorname{Re}=\frac{2 a d\left|\omega_{2}-\omega_{1}\right|}{(1+\eta) v}
$$

Легко показать, что справедливо соотношение

$$
\mathrm{Ta}=\sigma^{2} \mathrm{Re}^{2} .
$$

\section{Сравнение с экспериментами}

Результаты сравнения предсказаний модели обобщенного локального баланса с более ранними экспериментами для развитого турбулентного течения Тейлора-Куэтта были представлены в работах $[1,2]$.

На рис. 1. представлено сопоставление зависимостей безразмерного момента сил $G$, действующего на боковую поверхность вращающегося внутреннего цилиндра (внешний цилиндр покоится) от числа Рейнольдса $\mathrm{Re}_{1}$ по данным эксперимента [6] для отношения радиусов цилиндров $\eta=0.35$, и рассчитанной по уравнению (18). Это алгебраическое уравнение после деления обеих частей на величину $G_{1}$ решалось итеративным методом Ньютона-Рафсона. Можно говорить о сравнительно неплохом согласии теории и эксперимента, имея в виду отсутствие в теории дополнительных подгоночных констант для этого типа течения, кроме двух известных констант Прандтля-Кармана. На рис. 2 представлено сопоставление двух теоретических зависимостей величины от числа Тейлора Та, полученных путем решения алгебраического уравнения (10) методом Ньютона-Рафсона и асимптотической явной зависимостью (15), и экспериментальной зависимостью, полученной в работе [7]. Эта экспериментальная зависимость представлена также в работе [8], откуда и были перенесены данные на рис. 2.

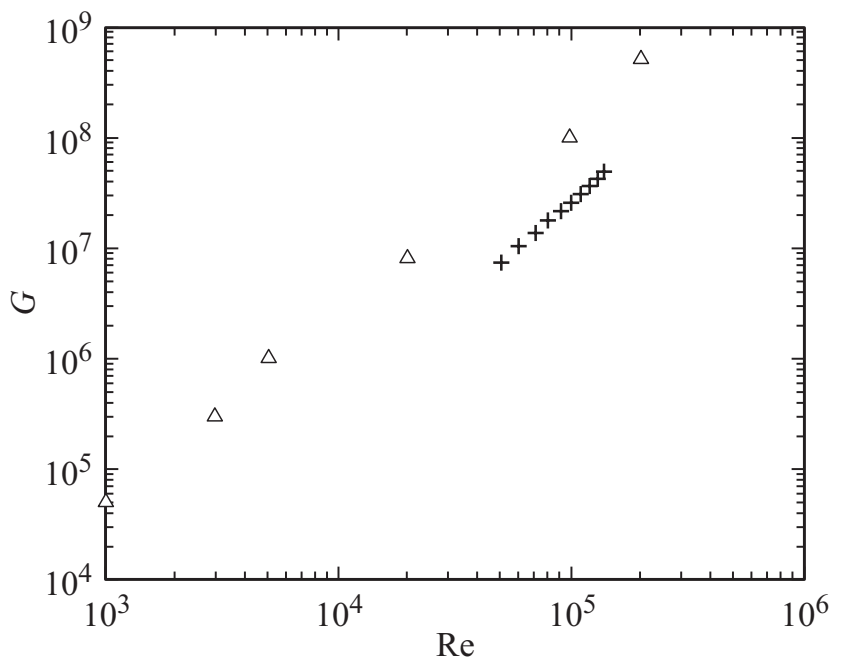

Pис. 1. Экспериментальные измерения (треугольники) [6] и решение уравнения $(10)(+)$ для определения безразмерного момента сил $G$ как функция числа Рейнольдса Re.

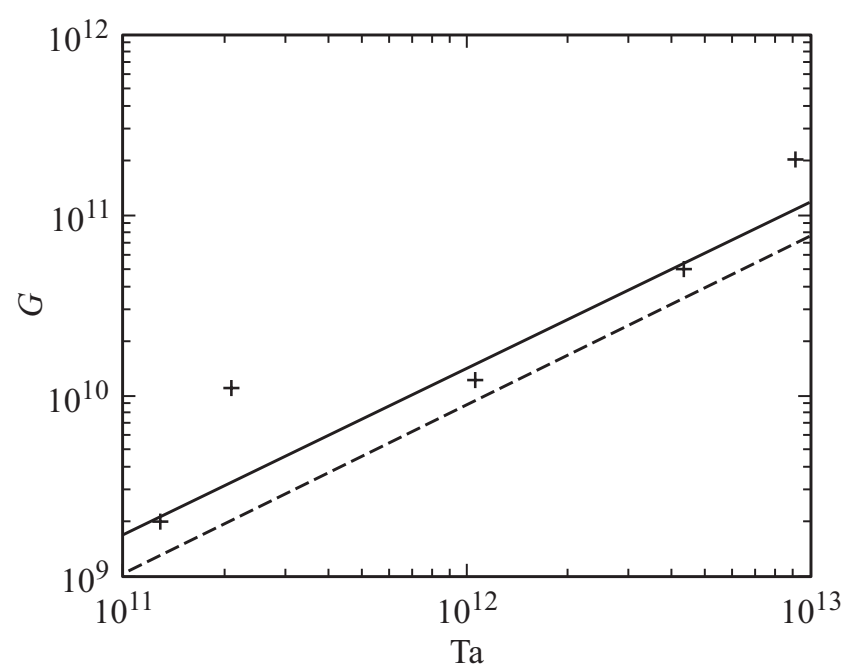

Рис. 2. Экспериментальные измерения [7] $(+)$, решения уравнения (10) (сплошная линия), формула (15) (штриховая линия) для определения безразмерного момента сил $G$ как функции числа Тейлора Та.

Переход от числа Рейнольдса Re к числу Тейлора Ta осуществлялся по формуле (30). Из-за размытости данных [7] (данные работы [3] еще более размыты, что видно из работы $[8]$ ), для сравнения была отобрана серия данных, соответствующая экстраполяции [7] к бесконечному числу Россби $\mathrm{Ro}_{\infty}$, где число Россби определяется как

$$
\operatorname{Ro}=\left(\omega_{1}-\omega_{2}\right) / \omega_{2}=\left(\operatorname{Re}_{1} / \eta \operatorname{Re}_{2}\right)-1 .
$$

Видно хорошее согласие обеих теоретических кривых и экспериментальных данных. Обсуждение имеющихся к настоящему моменту экспериментальных данных по течению Тейлора-Куэтта опубликовано в работе [9]. 


\section{Заключение}

В целом на наш взгляд, модель обобщенного локального баланса является одной из лучшей среди существующих теорий для развитого турбулентного течения Тейлора-Куэтта, что показывает сравнение предсказаний модели и экспериментами. Однако в рамках этой модели остаются нерешенные вопросы. Является ли размытость экспериментальных данных следствием дополнительной, детерминированной, более слабой зависимостью момента сил $G$ от соотношения частот вращения, как утверждается в экспериментальных работах $[3,7,8]$ ? Или это следствия детерминированного хаоса, являющегося неотъемлемой частью этого типа течения, и нужно привлекать для расчетов нестационарные уравнения модели [1]?

\section{Список литературы}

[1] Balonishnikov A.M. // Phys. Rev. E. 2000. Vol. 61. N 2. P. 1390-1394.

[2] Балонишников А.М. // ЖТФ 2003. Т. 73. Вып. 2. С. 139-140.

[3] Van Gils D.P.M., Huisman S.G., Bruggert G.-W., Sun C., Lohse D. // Phys. Rev. Lett. 2011. Vol. 106. P. 024502.

[4] Kraichnan R.H. // Phys. Fluids 1962. Vol. 5. P. 1374.

[5] Dubrulle B., Hersant F. // Eur. Phys. J. 2002. Vol. B26. P. 379.

[6] Burin M.J., Schartman E, Ji H. // Exp. Fluids. 2010. Vol. 48. P. 763.

[7] Paoletti M.S., Lathrop D.P. // Phys. Rev. Lett. 2011. Vol. 106. P. 024501.

[8] Paoletti M.S., van Gils DP.M., Dubrulle B., Chao Sun, Lohse D., Lathrop D.P. // Astronom. Astrophys. 2012. Vol. 547. A. 64

[9] Nordsiek F., Huisman S.G., van der Veen R.C.A., Chao Sun, Lohse D., Lathrop D.P. // J. Fluid Mech. 2015. Vol. 774. P. 342. 